\title{
POSITIVE PERIODIC SOLUTIONS OF A DISCRETE MUTUALISM MODEL WITH TIME DELAYS
}

\author{
YONGKUN LI
}

Received 23 August 2004 and in revised form 14 December 2004

A discrete periodic mutualism model with time delays is investigated. By using Gaines and Mawhin's continuation theorem of coincidence degree theory, the existence of positive periodic solutions of the model is established.

\section{Introduction}

Two species cohabit a common habitat and each species enhances the average growth rate of the other, this type of ecological interaction is known as facultative mutualism [8]. In [6], the author has studied the existence of positive periodic solutions of the periodic mutualism model

$$
\begin{aligned}
& \frac{\mathrm{d} N_{1}(t)}{\mathrm{d} t}=r_{1}(t) N_{1}(t)\left[\frac{K_{1}(t)+\alpha_{1}(t) N_{2}\left(t-\tau_{2}(t)\right)}{1+N_{2}\left(t-\tau_{2}(t)\right)}-N_{1}\left(t-\sigma_{1}(t)\right)\right], \\
& \frac{\mathrm{d} N_{2}(t)}{\mathrm{d} t}=r_{2}(t) N_{2}(t)\left[\frac{K_{2}(t)+\alpha_{2}(t) N_{1}\left(t-\tau_{1}(t)\right)}{1+N_{1}\left(t-\tau_{1}(t)\right)}-N_{2}\left(t-\sigma_{2}(t)\right)\right],
\end{aligned}
$$

where $r_{i}, K_{i}, \alpha_{i} \in C\left(\mathbb{R}, \mathbb{R}^{+}\right), \alpha_{i}>K_{i}, i=1,2, \tau_{i}, \sigma_{i} \in C\left(\mathbb{R}, \mathbb{R}^{+}\right), i=1,2, r_{i}, K_{i}, \alpha_{i}, \tau_{i}, \sigma_{i}(i=$ $1,2)$ are functions of period $\omega>0$. Since the study on periodic solutions of a population model is of great interest in mathematical biology [5] and many authors [1,7] have argued that the discrete-time models governed by difference equations are more appropriate than the continuous ones when the populations have nonoverlapping generations, then, discrete-time models can provide efficient computational types of continuous models for numerical simulations. It is reasonable to study the discrete-time mutualism model governed by difference equations.

One of the ways of deriving difference equations modeling the dynamics of populations with nonoverlapping generations is based on appropriate modifications of the corresponding models with overlapping generations $[2,4]$. In this approach, differential equations with piecewise constant arguments have been proved to be useful. Following the same idea and the same method in $[2,4]$, one can easily derive the following discrete 
analog of (1.1), which takes the form of

$$
\begin{aligned}
& x_{1}(k+1)=x_{1}(k) \exp \left[\frac{r_{1}(k) K_{1}(k)+r_{1}(k) \alpha_{1}(k) x_{2}\left(k-\tau_{2}(k)\right)}{1+x_{2}\left(k-\tau_{2}(k)\right)}-r_{1}(k) x_{1}\left(k-\sigma_{1}(k)\right)\right], \\
& x_{2}(k+1)=x_{2}(k) \exp \left[\frac{r_{2}(k) K_{2}(k)+r_{2}(k) \alpha_{2}(k) x_{1}\left(k-\tau_{1}(k)\right)}{1+x_{1}\left(k-\tau_{1}(k)\right)}-r_{2}(k) x_{2}\left(k-\sigma_{2}(k)\right)\right] .
\end{aligned}
$$

The exponential form of (1.2) is more biologically reasonable than that directly derived by replacing the differential by difference in (1.1). Our purpose in this paper is to use Mawhin's continuous theorem [3] to study the existence of positive periodic solutions of (1.2).

Let $\mathbb{Z}, \mathbb{R}$, and $\mathbb{R}^{2}$ denote the sets of all integers and two-dimensional Euclidean vector space, respectively. Throughout this paper, we always assume that the following hold.

$\left(\mathrm{H}_{1}\right)$ For $i=1,2, r_{i}, K_{i}, \alpha_{i}: \mathbb{Z} \rightarrow(0, \infty)$ and $\tau_{i}, \sigma_{i}: \mathbb{Z} \rightarrow[0, \infty)$ are all $\omega$-periodic, that is,

$$
\begin{gathered}
r_{i}(k+\omega)=r_{i}(k), \quad K_{i}(k+\omega)=K_{i}(k), \quad \alpha_{i}(k+\omega)=\alpha_{i}(k), \\
\tau_{i}(k+\omega)=\tau_{i}(k), \quad \sigma_{i}(k+\omega)=\sigma_{i}(k), \quad k \in \mathbb{Z} .
\end{gathered}
$$

$\left(\mathrm{H}_{2}\right)$ For $i=1,2, \alpha_{i}(k)>K_{i}(k), k \in \mathbb{Z}$.

\section{Existence of a positive periodic solution}

In order to use Mawhin's continuous theorem to establish the existence of at least one positive periodic solution of (1.2), we need to make some preparations.

Let $X, Y$ be normed vector spaces, let $L: \operatorname{Dom} L \subset X \rightarrow Y$ be a linear mapping, and let $N: X \rightarrow Y$ be a continuous mapping. The mapping $L$ will be called a Fredholm mapping of index zero if $\operatorname{dim} \operatorname{Ker} L=\operatorname{codim} \operatorname{Im} L<+\infty$ and $\operatorname{Im} L$ is closed in $Y$. If $L$ is a Fredholm mapping of index zero, there exist continuous projectors $P: X \rightarrow X$ and $Q: Y \rightarrow Y$ such that $\operatorname{Im} P=\operatorname{Ker} L, \operatorname{Ker} Q=\operatorname{Im} L=\operatorname{Im}(I-Q)$. It follows that the mapping $\left.L\right|_{\operatorname{Dom} L \cap \operatorname{Ker} P}$ : $(I-P) X \rightarrow \operatorname{Im} L$ is invertible. We denote the inverse of the mapping by $K_{P}$. If $\Omega$ is an open bounded subset of $X$, the mapping $N$ will be called $L$-compact on $\bar{\Omega}$ if $Q N(\bar{\Omega})$ is bounded and $K_{P}(I-Q) N: \bar{\Omega} \rightarrow X$ is compact. Since $\operatorname{Im} Q$ is isomorphic to $\operatorname{Ker} L$, there exists an isomorphism $J: \operatorname{Im} Q \rightarrow \operatorname{Ker} L$.

For convenience, we introduce Mawhin's continuous theorem [3, page 40] as follows.

Lemma 2.1. Let L be a Fredholm mapping of index zero and let $N$ be L-compact on $\bar{\Omega}$. Assume that

(i) for each $\lambda \in(0,1)$, every solution $x$ of $L x=\lambda N x$ is such that $x \notin \partial \Omega$;

(ii) $Q N x \neq 0$ for each $x \in \partial \Omega \bigcap \operatorname{Ker} L$ and

$$
\operatorname{deg}(J N Q, \Omega \bigcap \operatorname{Ker} L, 0) \neq 0 .
$$

Then the operator equation $L x=N x$ has at least one solution in $\bar{\Omega} \bigcap \operatorname{Dom} L$. 
In what follows, we will use the notations

$$
I_{\omega}=\{0,1, \ldots, \omega-1\}, \quad \bar{u}=\frac{1}{\omega} \sum_{k=0}^{\omega-1} u(k),
$$

where $\{u(k)\}$ is an $\omega$-periodic sequence of real numbers defined for $k \in \mathbb{Z}$.

The following result was given by [2, Lemma 3.2].

Lemma 2.2. Let $f: \mathbb{Z} \rightarrow \mathbb{R}$ be $\omega$-periodic, that is, $f(k+\omega)=f(k)$. Then for any fixed $k_{1}, k_{2} \in I_{\omega}$, and any $k \in \mathbb{Z}$,

$$
\begin{aligned}
& f(k) \leq f\left(k_{1}\right)+\sum_{s=0}^{\omega-1}|f(s+1)-f(s)|, \\
& f(k) \geq f\left(k_{2}\right)-\sum_{s=0}^{\omega-1}|f(s+1)-f(s)| .
\end{aligned}
$$

The following result was given by [6, Lemma 2.2].

Lemma 2.3. Let

$$
f(x, y)=\left(a_{1}-\frac{a_{1}-b_{1}}{1+e^{y}}-c_{1} e^{x}, a_{2}-\frac{a_{2}-b_{2}}{1+e^{x}}-c_{2} e^{y}\right)
$$

and $\Omega=\left\{(x, y)^{T} \in \mathbb{R}^{2}:|x|+|y|<M\right\}$, where $M, a_{i}, b_{i}, c_{i} \in \mathbb{R}^{+}$are constants, $a_{i}>b_{i}, i=$ 1,2 , and $M>\max \left\{\left|\ln \left(a_{i} / c_{i}\right)\right|,\left|\ln \left(b_{i} / c_{i}\right)\right|, i=1,2\right\}$. Then

$$
\operatorname{deg}\{f, \Omega,(0,0)\} \neq 0 .
$$

Now we state our fundamental theorem about the existence of a positive $\omega$-periodic solution of (1.2).

Theorem 2.4. Assume that $\left(H_{1}\right)$ and $\left(H_{2}\right)$ hold, then (1.2) has at least one positive $\omega$ periodic solution.

Proof. Consider the following system of difference equations with delays:

$$
\begin{aligned}
& y_{1}(k+1)-y_{1}(k)=r_{1}(k)\left[\frac{K_{1}(k)+\alpha_{1}(k) \exp \left\{y_{2}\left(k-\tau_{2}(k)\right)\right\}}{1+\exp \left\{y_{2}\left(k-\tau_{2}(k)\right)\right\}}-\exp \left\{y_{1}\left(k-\sigma_{1}(k)\right)\right\}\right], \\
& y_{2}(k+1)-y_{2}(k)=r_{2}(k)\left[\frac{K_{2}(k)+\alpha_{2}(k) \exp \left\{y_{1}\left(k-\tau_{1}(k)\right)\right\}}{1+\exp \left\{y_{1}\left(k-\tau_{1}(k)\right)\right\}}-\exp \left\{y_{2}\left(k-\sigma_{2}(k)\right)\right\}\right],
\end{aligned}
$$

where $r_{i}, K_{i}, \alpha_{i}, \tau_{i}, \sigma_{i}(i=1,2)$ are the same as those in (1.2). It is easy to see that if (2.6) has an $\omega$-periodic solution $\left\{\left(y_{1}^{*}(k), y_{2}^{*}(k)\right)^{T}\right\}$, then $\left\{\left(x_{1}^{*}(k), x_{2}^{*}(k)\right)^{T}\right\}=\left\{\left(\exp \left\{y_{1}^{*}(k)\right\}\right.\right.$, $\left.\left.\exp \left\{y_{2}^{*}(k)\right\}\right)^{T}\right\}$ is a positive $\omega$-periodic solution of (1.2). Therefore, to complete the proof, it suffices to show that system (2.6) has at least one $\omega$-periodic solution.

Define

$$
l_{2}=\left\{y=\{y(k)\}: y(k) \in \mathbb{R}^{2}, k \in \mathbb{Z}\right\} .
$$


Let $l^{\omega} \subset l_{2}$ denote the subspace of all $\omega$-periodic sequences equipped with the norm $\|\cdot\|$, that is,

$$
\begin{gathered}
\|y\|=\left\|\left(y_{1}, y_{2}\right)^{T}\right\|=\max _{k \in I_{\omega}}\left|y_{1}(k)\right|+\max _{k \in I_{\omega}}\left|y_{2}(k)\right|, \\
\text { for any } y=\left\{\left(y_{1}(k), y_{2}(k)\right), k \in \mathbb{Z}\right\} \in l^{\omega} .
\end{gathered}
$$

It is not difficult to show that $l^{\omega}$ is a finite-dimensional Banach space.

Let

$$
\begin{aligned}
& l_{0}^{\omega}=\left\{y=\{y(k)\} \in l^{\omega}: \sum_{k=0}^{\omega-1} y(k)=0\right\}, \\
& l_{c}^{\omega}=\left\{y=\{y(k)\} \in l^{\omega}: y(k)=h \in \mathbb{R}^{2}, k \in \mathbb{Z}\right\},
\end{aligned}
$$

then it is easy to check that $l_{0}^{\omega}$ and $l_{c}^{\omega}$ are both closed linear subspaces of $l^{\omega}$ and

$$
l^{\omega}=l_{0}^{\omega} \oplus l_{c}^{\omega}, \quad \operatorname{dim} l_{c}^{\omega}=2 .
$$

Take $X=Y=l^{\omega}$ and let

$$
\begin{aligned}
& (N y)(k) \\
& =\left[\begin{array}{l}
r_{1}(k)\left[\frac{K_{1}(k)+\alpha_{1}(k) \exp \left\{y_{2}\left(k-\tau_{2}(k)\right)\right\}}{1+\exp \left\{y_{2}\left(k-\tau_{2}(k)\right)\right\}}-\exp \left\{y_{1}\left(k-\sigma_{1}(k)\right)\right\}\right] \\
r_{2}(k)\left[\frac{K_{2}(k)+\alpha_{2}(k) \exp \left\{y_{1}\left(k-\tau_{1}(k)\right)\right\}}{1+\exp \left\{y_{1}\left(k-\tau_{1}(k)\right)\right\}}-\exp \left\{y_{2}\left(k-\sigma_{2}(k)\right)\right\}\right]
\end{array}\right], \quad y \in X, k \in \mathbb{Z}, \\
& (L y)(k)=y(k+1)-y(k), \quad y \in X, k \in \mathbb{Z},
\end{aligned}
$$

then it is easy to see that $L$ is a bounded linear operator with

$$
\operatorname{Ker} L=l_{c}^{\omega}, \quad \operatorname{Im} L=l_{0}^{\omega}, \quad \operatorname{dim} \operatorname{Ker} L=2=\operatorname{codim} \operatorname{Im} L,
$$

then it follows that $L$ is a Fredholm mapping of index zero.

Define

$$
P y=\frac{1}{\omega} \sum_{s=0}^{\omega-1} y(s), \quad y \in X, \quad Q z=\frac{1}{\omega} \sum_{s=0}^{\omega-1} z(s), \quad z \in Y .
$$

It is not difficult to show that $P$ and $Q$ are continuous projectors such that

$$
\operatorname{Im} P=\operatorname{Ker} L, \quad \operatorname{Im} L=\operatorname{Ker} Q=\operatorname{Im}(I-Q) .
$$

Furthermore, the generalized inverse (to $L$ ) $K_{P}: \operatorname{Im} L \rightarrow \operatorname{Ker} P \bigcap \operatorname{Dom} L$ exists, which is given by

$$
\left(K_{P} z\right)(n)=\sum_{i=0}^{n-1} z(i)-\frac{1}{\omega} \sum_{i=1}^{\omega} \sum_{s=0}^{i-1} z(s), \quad n \in \mathbb{Z} .
$$


Obviously, $Q N$ and $K_{P}(I-Q) N$ are continuous. Since $X$ is a finite-dimensional Banach space, one can easily show that $\overline{K_{P}(I-Q) N(\bar{\Omega})}$ is compact for any open bounded set $\Omega \subset X$. Moreover, $Q N(\bar{\Omega})$ is bounded, and hence $N$ is $L$-compact on $\bar{\Omega}$ with any open bounded set $\Omega \subset X$.

Now we are in a position to search for an appropriate open bounded subset $\Omega \subset X$ for the continuation theorem. Corresponding to the operator equation $L x=\lambda N x, \lambda \in(0,1)$, we have

$$
\begin{aligned}
& y_{1}(k+1)-y_{1}(k)=\lambda r_{1}(k)\left[\frac{K_{1}(k)+\alpha_{1}(k) \exp \left\{y_{2}\left(k-\tau_{2}(k)\right)\right\}}{1+\exp \left\{y_{2}\left(k-\tau_{2}(k)\right)\right\}}-\exp \left\{y_{1}\left(k-\sigma_{1}(k)\right)\right\}\right], \\
& y_{2}(k+1)-y_{2}(k)=\lambda r_{2}(k)\left[\frac{K_{2}(k)+\alpha_{2}(k) \exp \left\{y_{1}\left(k-\tau_{1}(k)\right)\right\}}{1+\exp \left\{y_{1}\left(k-\tau_{1}(k)\right)\right\}}-\exp \left\{y_{2}\left(k-\sigma_{2}(k)\right)\right\}\right] .
\end{aligned}
$$

Assume that $\left\{\left(y_{1}(k), y_{2}(k)\right)^{T}\right\} \in X$ is a solution of system (2.16) for a certain $\lambda \in(0,1)$. Summing on both sides of (2.16) from 0 to $\omega-1$ with respect to $k$, we obtain

$$
\begin{aligned}
& \sum_{k=0}^{\omega-1} r_{1}(k)\left[\frac{K_{1}(k)+\alpha_{1}(k) \exp \left\{y_{2}\left(k-\tau_{2}(k)\right)\right\}}{1+\exp \left\{y_{2}\left(k-\tau_{2}(k)\right)\right\}}-\exp \left\{y_{1}\left(k-\sigma_{1}(k)\right)\right\}\right]=0, \\
& \sum_{k=0}^{\omega-1} r_{2}(k)\left[\frac{K_{2}(k)+\alpha_{2}(k) \exp \left\{y_{1}\left(k-\tau_{1}(k)\right)\right\}}{1+\exp \left\{y_{1}\left(k-\tau_{1}(k)\right)\right\}}-\exp \left\{y_{2}\left(k-\sigma_{2}(k)\right)\right\}\right]=0 .
\end{aligned}
$$

It is easy to see that we can rewrite (2.17) and (2.18), respectively, as

$$
\begin{aligned}
& \sum_{k=0}^{\omega-1} \frac{r_{1}(k)\left(\alpha_{1}(k)-K_{1}(k)\right)}{1+\exp \left\{y_{2}\left(k-\tau_{2}(k)\right)\right\}}+\sum_{k=0}^{\omega-1} r_{1}(k) \exp \left\{y_{1}\left(k-\sigma_{1}(k)\right)\right\}=\sum_{k=0}^{\omega-1} r_{1}(k) \alpha_{1}(k), \\
& \sum_{k=0}^{\omega-1} \frac{r_{2}(k)\left(\alpha_{2}(k)-K_{2}(k)\right)}{1+\exp \left\{y_{1}\left(k-\tau_{1}(k)\right)\right\}}+\sum_{k=0}^{\omega-1} r_{2}(k) \exp \left\{y_{2}\left(k-\sigma_{2}(k)\right)\right\}=\sum_{k=0}^{\omega-1} r_{2}(k) \alpha_{2}(k) .
\end{aligned}
$$

Thus, from (2.16) and (2.19), it follows that

$$
\begin{aligned}
\sum_{k=0}^{\omega-1} \mid & y_{1}(k+1)-y_{1}(k) \mid \\
< & \lambda \sum_{k=0}^{\omega-1} r_{1}(k)\left[\frac{K_{1}(k)+\alpha_{1}(k) \exp \left\{y_{2}\left(k-\tau_{2}(k)\right)\right\}}{1+\exp \left\{y_{2}\left(k-\tau_{2}(k)\right)\right\}}+\exp \left\{y_{1}\left(k-\sigma_{1}(k)\right)\right\}\right] \\
< & \sum_{k=0}^{\omega-1} r_{1}(k) \alpha_{1}(k)+\sum_{k=0}^{\omega-1} \frac{r_{1}(k)\left(\alpha_{1}(k)-K_{1}(k)\right)}{1+\exp \left\{y_{2}\left(k-\tau_{2}(k)\right)\right\}} \\
& +\sum_{k=0}^{\omega-1} r_{1}(k) \exp \left\{y_{1}\left(k-\sigma_{1}(k)\right)\right\} \\
= & 2 \sum_{k=0}^{\omega-1} r_{1}(k) \alpha_{1}(k):=M_{1},
\end{aligned}
$$


504 Periodic solutions of a discrete mutualism model

that is,

$$
\sum_{k=0}^{\omega-1}\left|y_{1}(k+1)-y_{1}(k)\right|<M_{1}
$$

In a similar way, by (2.16) and (2.20), we have

$$
\sum_{k=0}^{\omega-1}\left|y_{2}(k+1)-y_{2}(k)\right|<2 \sum_{k=0}^{\omega-1} r_{2}(k) \alpha_{2}(k):=M_{2} .
$$

Moreover, from (2.19), it follows that

$$
\sum_{k=0}^{\omega-1} r_{1}(k) \alpha_{1}(k) \geq \sum_{k=0}^{\omega-1} r_{1}(k) \exp \left\{y_{1}\left(k-\sigma_{1}(k)\right)\right\} \geq \sum_{k=0}^{\omega-1} r_{1}(k) K_{1}(k),
$$

hence

$$
\frac{r_{1}^{*} \alpha_{1}^{*}}{\underline{r}_{1}} \omega \geq \sum_{k=0}^{\omega-1} \exp \left\{y_{1}\left(k-\sigma_{1}(k)\right)\right\} \geq \frac{\underline{r}_{1} \underline{K}_{1}}{r_{1}^{*}} \omega
$$

which implies that there exist points $k_{1}^{\prime}, k_{2}^{\prime} \in I_{\omega}$ such that

$$
\begin{aligned}
& y_{1}\left(k_{1}^{\prime}-\sigma_{1}\left(k_{1}^{\prime}\right)\right) \leq \ln \left[\frac{r_{1}^{*} \alpha_{1}^{*}}{\underline{r}_{1}}\right]:=C_{1}, \\
& y_{1}\left(k_{2}^{\prime}-\sigma_{1}\left(k_{2}^{\prime}\right)\right) \geq \ln \left[\frac{\underline{r}_{1} \underline{K}_{1}}{r_{1}^{*}}\right]:=C_{2},
\end{aligned}
$$

where $r_{1}^{*}=\max \left\{r_{1}(k), k \in I_{\omega}\right\}, \alpha_{1}^{*}=\max \left\{\alpha_{1}(k), k \in I_{\omega}\right\}, \underline{r}_{1}=\min \left\{r_{1}(k), k \in I_{\omega}\right\}, \underline{K}_{1}=$ $\min \left\{K_{1}(k), k \in I_{\omega}\right\}$. Denote $k_{i}^{\prime}-\sigma_{1}\left(k_{i}^{\prime}\right)=k_{i}+n_{i} \omega, k_{i} \in I_{\omega}$ and $n_{i}$ is an integer, $i=1,2$. Then

$$
y_{1}\left(k_{1}\right) \leq C_{1}, \quad y_{1}\left(k_{2}\right) \geq C_{2} .
$$

Similarly, by (2.20), we can obtain that there exist points $k_{3}, k_{4} \in I_{\omega}$ such that

$$
y_{2}\left(k_{3}\right) \leq \ln \left[\frac{r_{2}^{*} \alpha_{2}^{*}}{\underline{r}_{2}}\right]:=C_{3}, \quad y_{2}\left(k_{4}\right) \geq \ln \left[\frac{\underline{r}_{2} \underline{K}_{2}}{r_{2}^{*}}\right]:=C_{4},
$$

where $r_{2}^{*}=\max \left\{r_{2}(k), k \in I_{\omega}\right\}, \alpha_{2}^{*}=\max \left\{\alpha_{2}(k), k \in I_{\omega}\right\}, \underline{r}_{2}=\min \left\{r_{2}(k), k \in I_{\omega}\right\}$, and $\underline{K}_{2}=\min \left\{K_{2}(k), k \in I_{\omega}\right\}$.

Therefore, in view of (2.22)-(2.27) and Lemma 2.2, we have

$$
\begin{aligned}
& y_{1}(k) \leq y_{1}\left(k_{1}\right)+\sum_{s=0}^{\omega-1}\left|y_{1}(s+1)-y_{1}(s)\right|<C_{1}+M_{1}, \\
& y_{1}(k) \geq y_{1}\left(k_{2}\right)-\sum_{s=0}^{\omega-1}\left|y_{1}(s+1)-y_{1}(s)\right|>C_{2}-M_{1} .
\end{aligned}
$$


Hence,

$$
\max _{k \in I_{\omega}}\left|y_{1}(k)\right|<\max \left\{\left|C_{1}+M_{1}\right|,\left|C_{2}-M_{1}\right|\right\}:=A_{1}
$$

Similarly, it follows from (2.23)-(2.28) and Lemma 2.2 that

$$
\max _{k \in I_{\omega}}\left|y_{2}(k)\right|<\max \left\{\left|C_{3}+M_{2}\right|,\left|C_{4}-M_{2}\right|\right\}:=A_{2}
$$

Clearly, $A_{1}$ and $A_{2}$ are independent of $\lambda$. Denote $M=A_{1}+A_{2}+D$, where $D>0$ is taken sufficiently large such that $M>\max \left\{\left|\ln \left(a_{i} / c_{i}\right)\right|,\left|\ln \left(b_{i} / c_{i}\right)\right|, i=1,2\right\}$. Now we take $\Omega=$ $\{y=\{y(k)\} \in X:\|y\|<M\}$. This satisfies condition (i) in Lemma 2.1. When $y=$ $\left\{\left(y_{1}, y_{2}\right)^{T}\right\} \in \partial \Omega \cap \operatorname{Ker} L=\partial \Omega \cap \mathbb{R}^{2},\left(y_{1}, y_{2}\right)^{T}$ is a constant vector in $\mathbb{R}^{2}$ with $\left|y_{1}\right|+\left|y_{2}\right|=$ M. Then

$$
Q N\left[\begin{array}{l}
y_{1} \\
y_{2}
\end{array}\right]=\left[\begin{array}{l}
\overline{r_{1} \alpha_{1}}-\frac{\overline{r_{1} \alpha_{1}}-\overline{r_{1} K_{1}}}{1+e^{y_{2}}}-\bar{r}_{1} e^{y_{1}} \\
\overline{r_{2} \alpha_{2}}-\frac{\overline{r_{2} \alpha_{2}}-\overline{r_{2} K_{2}}}{1+e^{y_{1}}}-\bar{r}_{2} e^{y_{2}}
\end{array}\right] \neq\left[\begin{array}{l}
0 \\
0
\end{array}\right] .
$$

Furthermore, by Lemma 2.3, we have

$$
\operatorname{deg}\left\{J Q N\left(y_{1}, y_{2}\right)^{T}, \Omega,(0,0)\right\} \neq 0,
$$

where the degree is Brouwer degree and the isomorphism $J$ can be chosen to be the identity mapping, since $\operatorname{Im} Q=\operatorname{Ker} L$. By now we know that $\Omega$ verifies all the requirements in Lemma 2.1 and then (2.6) has at least one $\omega$-periodic solution. Therefore, (1.2) has at least one positive $\omega$-periodic solution. The proof is complete.

\section{Acknowledgment}

This work is supported by the National Natural Sciences Foundation of China under Grant 10361006 and the Natural Sciences Foundation of Yunnan Province under Grant 2003A0001M.

\section{References}

[1] R. P. Agarwal, Difference Equations and Inequalities: Theory, Methods and Applications, Monographs and Textbooks in Pure and Applied Mathematics, vol. 228, Marcel Dekker, New York, 2000.

[2] M. Fan and K. Wang, Periodic solutions of a discrete time nonautonomous ratio-dependent predator-prey system, Math. Comput. Modelling 35 (2002), no. 9-10, 951-961.

[3] R. E. Gaines and J. L. Mawhin, Coincidence Degree, and Nonlinear Differential Equations, Springer, Berlin, 1977.

[4] K. Gopalsamy, Stability and Oscillations in Delay Differential Equations of Population Dynamics, Mathematics and Its Applications, vol. 74, Kluwer Academic Publishers, Dordrecht, 1992.

[5] Y. Kuang, Delay Differential Equations with Applications in Population Dynamics, Mathematics in Science and Engineering, vol. 191, Academic Press, Massachusetts, 1993.

[6] Y. Li, On a periodic mutualism model, ANZIAM J. 42 (2001), no. 4, 569-580. 


\section{Periodic solutions of a discrete mutualism model}

[7] J. D. Murray, Mathematical Biology, Biomathematics, vol. 19, Springer, Berlin, 1989.

[8] C. L. Wolin and L. R. Lawlor, Models of facultative mutualism: density effects, Amer. Natural. 144 (1984), 843-862.

Yongkun Li: Department of Mathematics, Yunnan University, Kunming, Yunnan 650091, China E-mail address: yklie@ynu.edu.cn 


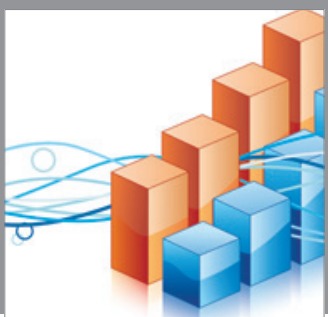

Advances in

Operations Research

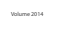

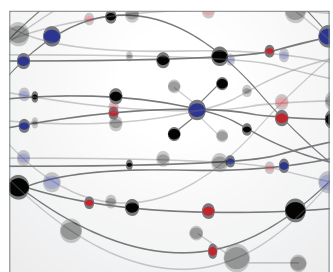

\section{The Scientific} World Journal
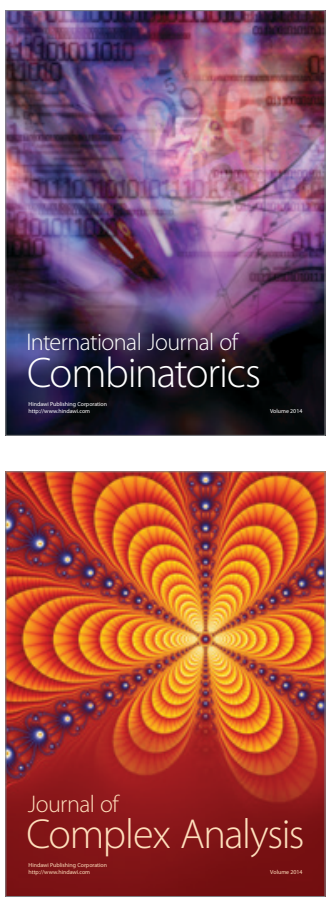

International Journal of

Mathematics and

Mathematical

Sciences
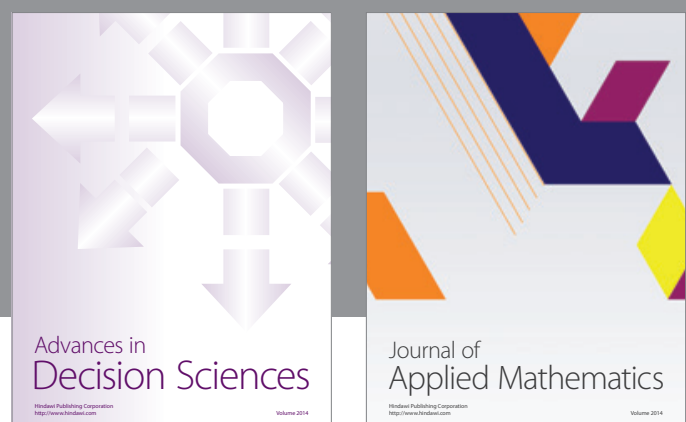

Journal of

Applied Mathematics
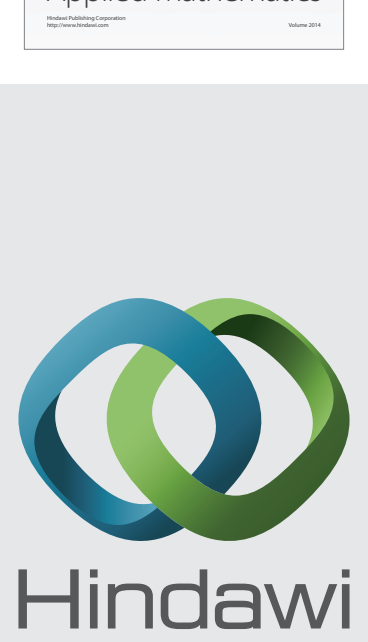

Submit your manuscripts at http://www.hindawi.com
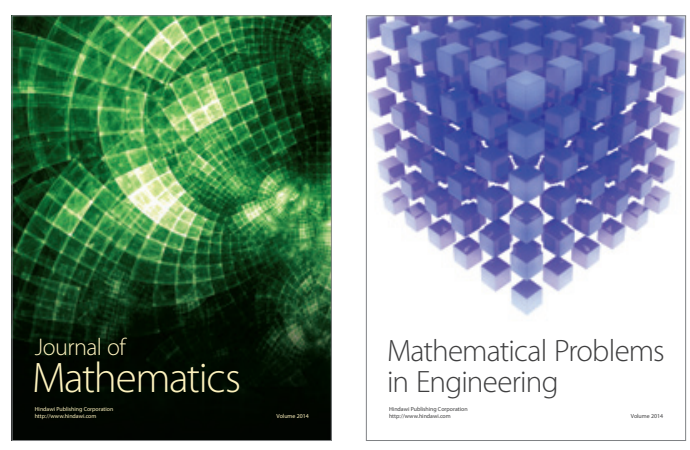

Mathematical Problems in Engineering
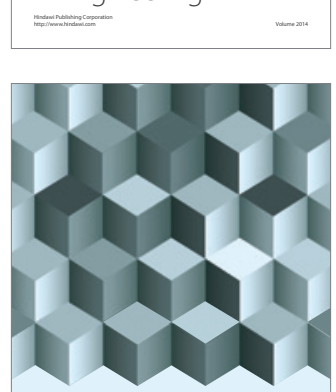

Journal of

Function Spaces
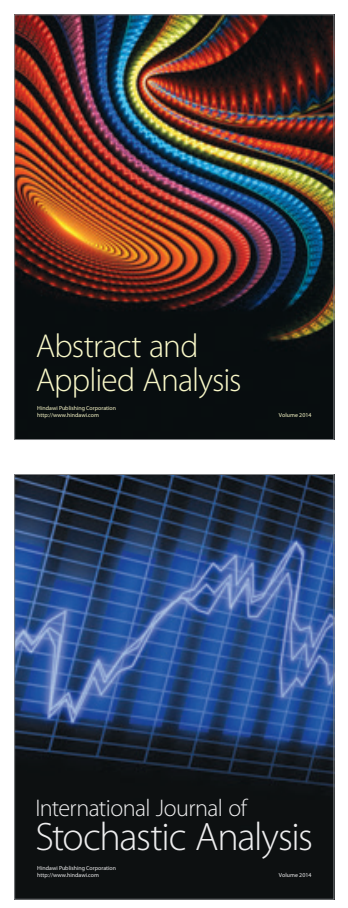

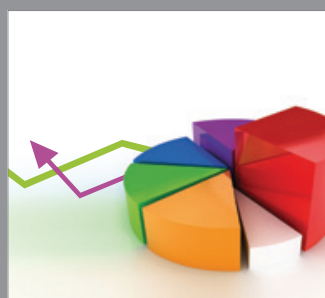

ournal of

Probability and Statistics

Promensencen
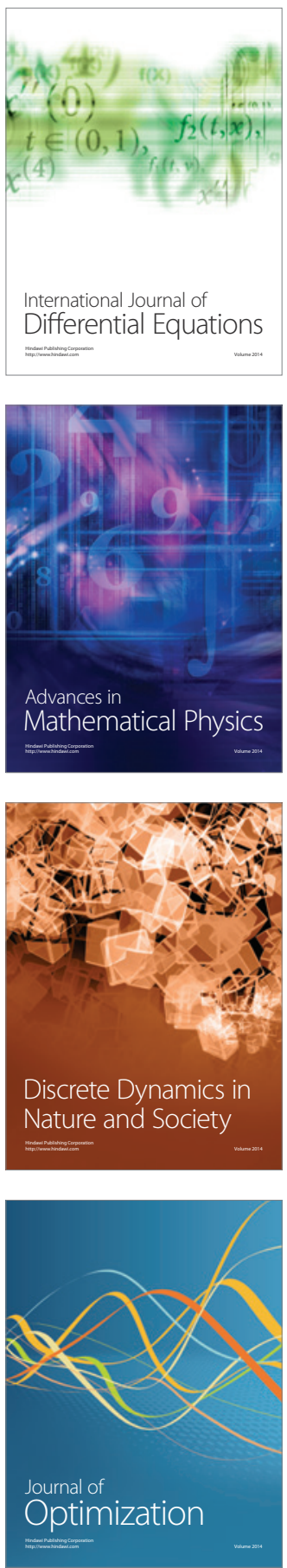